\title{
Geometry of faithful entanglement
}

\author{
Otfried Gühne, ${ }^{*}$ Yuanyuan Mao, ${ }^{\dagger}$ and Xiao-Dong $\mathrm{Yu}^{\ddagger}$ \\ Naturwissenschaftlich-Technische Fakultät, Universität Siegen, Walter-Flex-Straße 3, 57068 Siegen, Germany
}

(Dated: 17th March 2021)

\begin{abstract}
A typical concept in quantum state analysis is based on the idea that states in the vicinity of some pure entangled state share the same properties; implying that states with a high fidelity must be entangled. States whose entanglement can be detected in this way are also called faithful. We prove a structural result on the corresponding fidelity-based entanglement witnesses, resulting in a simple condition for faithfulness of a two-party state. For the simplest case of two qubits faithfulness can directly be decided and for higher dimensions accurate analytical criteria are given. Finally, our results show that faithful entanglement is, in a certain sense, useful entanglement; moreover, they establish connections to computational complexity and simplify several results in entanglement theory.
\end{abstract}

Introduction.- The certification and benchmarking of quantum devices is a fundamental problem in quantum information processing [1, 2]. Here, the exponentially increasing dimensionality of large quantum systems makes a full characterization usually impossible. Hence, one often restricts the attention to simple parameters, such as the average gate fidelity of a quantum gate [3,4], in order to compare different approaches or implementations. For the justification of such restrictions it is necessary, however, to know which phenomena are captured by the considered parameter and which not.

For the certification of quantum states, the fidelity of the observed state with a desired target state is often used as a benchmarking parameter. This can indeed be measured or estimated with relatively simple methods $[5,6]$. A natural question is then how this parameter is connected to the entanglement in the quantum state under scrutiny. This is relevant since entanglement is a key ingredient for applications like quantum key distribution [7] or quantum metrology [8].

A typical method to connect entanglement to the fidelity is based on the idea that states close to some known entangled pure state $|\psi\rangle$ must be entangled, too. Formally, the fidelity can be seen as a kind of distance of a general state $\varrho$ to the state $|\psi\rangle$, given by $F_{\psi}=\langle\psi|\varrho| \psi\rangle$. Then, one can formulate the resulting criteria also in the form of entanglement witnesses. That is, one considers the observable

$$
\mathcal{W}=\alpha \mathbb{1}-|\psi\rangle\langle\psi|
$$

In general, an entanglement witness is an observable which has a positive mean value on all separable states, hence a negative mean value signals the presence of entanglement [9-11]. Concretely, if this observable $\mathcal{W}$ is measured, one obtains $\operatorname{Tr}(\varrho \mathcal{W})=\alpha-F_{\psi}$, so if $F_{\psi}$ is above the threshold value $\alpha$, then the witness detects some entanglement. The fidelity-based witness in Eq. (1) is easy to construct and can be generalized to the multiparticle case. Note, however, that many other approaches to construct witnesses beyond the fidelity approach exist [12-17].
So, it is a central question to ask which quantum states can be detected as entangled using the fidelity and what are their physical properties. In Ref. [18], the authors coined the term faithful for states whose entanglement can be characterized using the fidelity, and provided first steps in distinguishing faithful and unfaithful states. For instance, an approach using convex optimization was provided which can prove the unfaithfulness of a state, with this one can show that in higher dimensional systems most states are unfaithful. Very recently, the phenomenon of faithfulness was also studied from an experimental point of view $[19,20]$.

In this paper we go further and deliver analytical results on faithful and unfaithful states. First, we show that one can restrict the attention to a special subclass of fidelity-based criteria. Then, we can derive some general results on faithful states: For two qubits, the faithfulness can directly be decided, and for higherdimensional systems, an analytical sufficient criterion for unfaithfulness can be given. We also show that faithful entanglement is useful entanglement, in the sense that it leads to a violation of Bell inequalities on multiple copies of a state. Moreover, our results simplify estimates of entanglement measures and we demonstrate that entanglement detection using the fidelity of a general projector $\Pi$ instead of a pure state $|\psi\rangle\langle\psi|$ is fundamentally different. Finally, we establish a connection to the problem of unitary quadratic minimization in complexity theory, as well as possible extensions to the Schmidt number classification of quantum states.

Entanglement, separability and witnesses. - We consider two parties, Alice and Bob, each of which owns a $d$ dimensional quantum system. In general, a mixed state is called separable, if it can be written as a convex combination of product states, $\varrho_{A B}=\sum_{k} p_{k}\left|a_{k}, b_{k}\right\rangle\left\langle a_{k}, b_{k}\right|$, where the $p_{k}$ form a probability distribution, that is, they are positive and sum up to one. States that can not be expressed in this way are called entangled.

There are many criteria for proving entanglement or separability of a given quantum state, although none of them solves the problem completely $[9,10]$. An important criterion is entanglement witnesses, which are 
observables that have a positive expectation value on all separable states, and a negative expectation value on some entangled states. It can be straightforwardly shown that, in principle, any entangled state can be detected by some suitable witness, but the problem is to construct all witnesses. This is, in fact, not easy and of the same complexity as the separability problem.

As already mentioned, one possible witness construction makes use of the fact that in the vicinity of an entangled pure state there are only entangled states. So, one can define a witness via $\mathcal{W}=\alpha \mathbb{1}-|\psi\rangle\langle\psi|$, where $\alpha$ is the maximal squared overlap between $|\psi\rangle$ and the separable states. This can be computed as $\alpha=$ $\sup _{|a, b\rangle}|\langle a, b \mid \psi\rangle|^{2}=s_{1}^{2}$, where the $s_{i}$ for $i=1, \ldots, r$ are the decreasingly ordered nonzero Schmidt coefficients of the state $|\psi\rangle$ in its Schmidt decomposition $|\psi\rangle=$ $\sum_{i=1}^{r} s_{i}|i i\rangle$ [21]. Here, the number of terms $r$ is called the Schmidt rank of the state. The smallest possible $\alpha$ occurs if the state $|\psi\rangle$ is a maximally entangled state, for instance $|\psi\rangle=\left|\phi^{+}\right\rangle=\sum_{i=1}^{d}|i i\rangle / \sqrt{d}$. Then we have

$$
\mathcal{W}=\frac{\mathbb{1}}{d}-\left|\phi^{+}\right\rangle\left\langle\phi^{+}\right|
$$

Clearly, one may also take other maximally entangled states which are locally equivalent to $\left|\phi^{+}\right\rangle$. For reasons that become apparent later, we call this set of witnesses of the type in Eq. (2) the relevant fidelity witnesses (RFW).

Main result.- We can directly state and prove the first main result:

Observation 1. Let $\varrho_{A B}$ be a faithful entangled state, i.e. its entanglement can be detected by some fidelity-based en- tanglement witness. Then $\varrho_{A B}$ can be detected by a relevant fidelity-based entanglement witness. In other words, a state $\varrho_{A B}$ is faithful if and only if there are local unitary transformations $U_{A}$ and $U_{B}$ such that

$$
\left\langle\phi^{+}\left|U_{A} \otimes U_{B} \varrho_{A B} U_{A}^{\dagger} \otimes U_{B}^{\dagger}\right| \phi^{+}\right\rangle>\frac{1}{d} .
$$

In order to present the idea of the proof we consider the case $d=4$, the general case is discussed in Appendix A. Let us assume that the fidelity-based witness detecting $\varrho_{A B}$ is given by $\mathcal{W}=s_{1}^{2} \mathbb{1}-|\psi\rangle\langle\psi|$, with $|\psi\rangle=\sum_{i=1}^{4} s_{i}|i i\rangle$. We consider eight RFWs, coming from the eight maximally entangled states

$$
\left|\phi_{\vec{a}}\right\rangle=\frac{1}{2}\left(|11\rangle+a_{2}|22\rangle+a_{3}|33\rangle+a_{4}|44\rangle\right),
$$

where $\vec{a}=\left(a_{2}, a_{3}, a_{4}\right)$ and the coefficients $a_{j}$ can have all possible values \pm 1 . This leads to eight RFWs $\mathcal{W}_{\vec{a}}=$ $\mathbb{1} / 4-\left|\phi_{\vec{a}}\right\rangle\left\langle\phi_{\vec{a}}\right|$.

We aim to show that one can find probabilities $p_{\vec{a}}$ such that the operator

$$
\mathcal{Z}=\mathcal{W}-4 s_{1}^{2} \sum_{\vec{a}} p_{\vec{a}} \mathcal{W}_{\vec{a}} \geq 0
$$

is positive semidefinite. This would prove already the main claim, since then $\operatorname{Tr}\left(\varrho_{A B} \mathcal{W}\right)<0$ implies that for at least one $\vec{a}$ we have $\operatorname{Tr}\left(\varrho_{A B} \mathcal{W}_{\vec{a}}\right)<0$. In order to prove Eq. (5) we aim to find $p_{\vec{a}}$ such that $\mathcal{Z}$ is diagonal. This is sufficient for positivity, as the diagonal entries of $\mathcal{Z}$ are independent of the $p_{\vec{a}}$. They are either zero or of the type $s_{1}^{2}-s_{j}^{2} \geq 0$, which ensures $\mathcal{Z} \geq 0$.

Looking at the relevant off-diagonal entries, one finds that for making $\mathcal{Z}$ diagonal, we have to find $p_{\vec{a}}$ such that

$$
\left(\begin{array}{cccc}
\times & \alpha_{2} & \alpha_{3} & \alpha_{4} \\
\alpha_{2} & \times & \alpha_{2} \alpha_{3} & \alpha_{2} \alpha_{4} \\
\alpha_{3} & \alpha_{2} \alpha_{3} & \times & \alpha_{3} \alpha_{4} \\
\alpha_{4} & \alpha_{2} \alpha_{4} & \alpha_{3} \alpha_{4} & \times
\end{array}\right)=\sum_{\vec{a}} p_{\vec{a}}\left(\begin{array}{cccc}
\times & a_{2} & a_{3} & a_{4} \\
a_{2} & \times & a_{2} a_{3} & a_{2} a_{4} \\
a_{3} & a_{2} a_{3} & \times & a_{3} a_{4} \\
a_{4} & a_{2} a_{4} & a_{3} a_{4} & \times
\end{array}\right)
$$

where we defined $\alpha_{i}=s_{i} / s_{1} \in[0,1]$. With a physical argument one can see now that a decomposition as in Eq. (6) can be found: One can view the three $\alpha_{i}$ as expectation values of some observables (say, $\sigma_{z}$ ) on a three-qubit product state $\varrho=\varrho_{A} \otimes \varrho_{B} \otimes \varrho_{C}$. The terms $\alpha_{i} \alpha_{j}$ correspond then to a two-body correlation $\left\langle\sigma_{z} \otimes \sigma_{z}\right\rangle$ on the same state. On the other hand, the right-hand side of Eq. (6) can be seen as a local hidden variable model, where the index $\vec{a}$ is the hidden variable occurring with probability $p_{\vec{a}}$, and the $a_{i}$ are the deterministic assignments for the measurement results of $\sigma_{z}$ on the different particles. For fully separable states, how- ever, it is well known that all measurements can be explained by a local hidden variable model [22], so there must be $p_{\vec{a}}$ such that Eq. (6) is fulfilled.

General implications. - First, from Observation 1 it is immediately clear that already for two qubits not all entangled states are faithful. The reason is the following: The RFW in Eq. (2) can be seen as a witnesses for the computable cross-norm or realignment (CCNR) criterion $[23,24]$. This follows from the fact that one may 
write it as

$$
\mathcal{W}=\frac{1}{d}\left(\mathbb{1}-\sum_{k=1}^{d^{2}} G_{k} \otimes G_{k}^{T}\right)
$$

where the $\left\{G_{k}\right\}$ form an orthogonal basis of the operator spaces for Alice and Bob and $(\cdot)^{T}$ denotes the transposition [15]. This means that only states that violate the CCNR criterion can be faithful. But it is known that already for two qubits there are entangled states that do not violate this criterion [25].

Second, using the Jamiołkowski isomorphism [26, 27], one can directly see that the RFW in Eq. (2) corresponds to the reduction map. From this one has directly the already known result [16] that only the entanglement in states which violate the reduction criterion can be detected by the fidelity. This further implies that only states that violate the criterion of the positivity of the partial transpose (PPT) can be faithful.

Third, the left hand side of the inequality (3) is related to a well-known quantity called the singlet fraction. This concept was introduced in Ref. [28] in a slightly more general form, where one tries to maximize the fidelity with the maximally entangled state, using trace-preserving local quantum operations and classical communication. Then, it was proved that having a singlet fraction larger than $1 / d$ is a necessary and sufficient condition for a state to offer a quantum advantage in teleportation. It was also shown that for every state $\varrho$ with singlet fraction larger than $1 / d$, there exists a number of copies $k$ of $\varrho$ such that $\varrho^{\otimes k}$ is nonlocal [29]. Together with these results, Observation 1 implies that faithful states are useful for quantum teleportation and that multiple copies of them violate a Bell inequality. This shows that fidelity-based entanglement witnesses detect entanglement that is useful.

Finally, the connection to the singlet fraction delivers more insights: For two qubits this quantity can be lower bounded by the entanglement as quantified by the concurrence or negativity [30]. From these results one can directly conclude that if the concurrence exceeds $1 / 2$ or the negativity exceeds $(\sqrt{2}-1) / 2$, then the state is faithful. Also, Ref. [31] implies that any two-qubit state is faithful after suitable local filtering operations. Moreover, as we elaborate in Appendix B, the unitary optimization in Eq. (3) is directly linked to the problem of unitary quadratic minimization, which was recently shown to be NP-hard [32].

Deciding faithfulness for qubits. - The problem of maximizing the overlap with a maximally entangled state in some basis for two qubits has been solved before [33], but in order to generalize it later, we formulate it in a different language. We can decompose a general two- qubit state $\varrho_{A B}$ into Pauli matrices,

$$
\varrho_{A B}=\frac{1}{4} \sum_{i, j=0}^{3} \lambda_{i j} \sigma_{i} \otimes \sigma_{j},
$$

where $\lambda_{i j}=\operatorname{Tr}\left(\varrho \sigma_{i} \otimes \sigma_{j}\right)$ and $\sigma_{0}=\mathbb{1}$ denotes the identity matrix. Then we consider the operator

$$
X_{d}\left(\varrho_{A B}\right)=\varrho_{A B}-\frac{1}{d}\left(\varrho_{A} \otimes \mathbb{1}+\mathbb{1} \otimes \varrho_{B}\right)+\frac{2}{d^{2}} \mathbb{1} \otimes \mathbb{1},
$$

where, for the case of two qubits, we take $d=2$. In terms of the representation in Eq. (8) this operator has a blockdiagonal $\lambda_{i j}$, the terms $\lambda_{i 0}$ and $\lambda_{0 j}$ for $i, j=1,2,3$ corresponding to the marginals have been removed. Note that we have for any maximally entangled state in any basis $\left\langle\phi^{+}\left|X_{2}(\varrho)\right| \phi^{+}\right\rangle=\left\langle\phi^{+}\left|\varrho_{A B}\right| \phi^{+}\right\rangle$, as for maximally entangled states the marginals are maximally mixed.

By local unitary transformations we can diagonalize the remaining $3 \times 3$ matrix $\lambda_{i j}$ for $i, j=1,2,3$ for the operator $X_{2}$. In this basis, $X_{2}$ is Bell diagonal, and we can directly read of the maximal overlap with Bell states by computing the maximal eigenvalue. We can summarize:

Observation 2. A two-qubit state $\varrho_{A B}$ is faithful if and only if the maximal eigenvalue of $X_{2}\left(\varrho_{A B}\right)$ in $E q$. (9) is larger than $1 / 2$.

Deciding faithfulness for higher dimensions. - First, we consider $X_{d}\left(\varrho_{A B}\right)$ from above, and if its largest eigenvalue is smaller than $1 / d$, the state cannot be faithful. The proof of this statement follows along the same lines as for qubits, one just needs to replace the Pauli matrices by some other basis of the operator space, where one basis element is proportional to the identity and the other elements are traceless. Then, the block of the matrix $\lambda$ can, in general, not be diagonalized anymore. Still, the maximal eigenvalue of $X_{d}$ is an upper bound on the overlap with maximally entangled states.

Alternatively, instead of maximizing the overlap over all maximally entangled states, one can maximize the overlap over all states with maximally mixed reduced states. That is, one considers the relaxed optimization

$$
\begin{aligned}
\text { max: } & \operatorname{Tr}\left(\varrho_{A B} \chi\right), \\
\text { subject to: } & \operatorname{Tr}(\chi)=1, \quad \chi \geq 0, \\
& \operatorname{Tr}_{A}(\chi)=\operatorname{Tr}_{B}(\chi)=\frac{\mathbb{1}}{d} .
\end{aligned}
$$

This is a simple SDP, and if a result smaller than $1 / d$ is found, the state cannot be faithful. In fact, one can show that the dual of this SDP is equivalent to the SDP2 presented in Ref. [18].

The formulation in Eq. (10) has, however, two advantages. First it can also be used to prove that a state is faithful. If the SDP returns a value larger than $1 / d$ one can check whether the optimal $\chi$ is a pure state. If this is the case, this state is maximally entangled and $\varrho_{A B}$ must be faithful. Second, one can systematically improve the 
SDP in Eq. (10) by adding rank constraints on $\chi$. These can be implemented by a hierarchy of SDPs [34] and detect indeed more states; see also below. We summarize:

Observation 3. Consider a general two-qudit state $\varrho_{A B}$. (a) If the largest eigenvalue of the operator $X_{d}\left(\varrho_{A B}\right)$ in Eq. (9) is not larger than $1 / d$, then $\varrho_{A B}$ is unfaithful. (b) If the semidefinite program from Eq. (10) has an optimal value not larger than $1 / d$, then $\varrho_{A B}$ is unfaithful. (c) If the optimization in Eq. (10) returns a value larger than $1 / d$ and the optimal $\chi$ is a pure state, then $\varrho_{A B}$ is faithful.

We add that the condition 3(b) detects strictly more states as unfaithful than condition 3(a). The reason is that in Eq. (10) one can directly replace $\varrho_{A B}$ with $X_{d}$ from Eq. (9), without changing the result of the SDP. Then, the optimization can further be relaxed by considering only the largest eigenvalue of $X_{d}$.

Numerical studies. - Armed with these insights, we generated random states in the Bures metric to estimate the fraction of the unfaithful states [35], additional results for the Hilbert-Schmidt distribution are described in Appendix C. For two qubits, we generated $10^{6}$ states and found $7.32 \%$ of all states separable via the condition of the positivity of the partial transpose (PPT) [9] and $15.44 \%$ entangled, but unfaithful. The remaining $77.24 \%$ are faithful. These values coincide up to statistical fluctuations with the values reported for the sufficient criterion SDP2 for unfaithfulness in Ref. [18] (or Eq. (10)). Indeed, using the fact that for $d=2$ the extreme points of the unital maps are unitary maps [36], one can see that in this case the extreme points of the matrices $\chi$ considered in Eq. (10) are the projectors onto maximally entangled states, hence for $d=2$ the SDP2 in Ref. [18] or Observation 3(b) are necessary and sufficient for faithfulness.

For higher dimensions the results are given in Table I. In the underlying samples of states it turned out that any state not obeying the criterion in Observation 3(b) is faithful. This, however, is not generally true. Consider a highly entangled state $\varrho_{A B}$, where the relaxed optimization in Eq. (10) gives a strictly larger value than the optimization of the overlap with all maximally entangled state, see Eq. (3), but both values are larger than $1 / d$. Such states can be found by random search. Then, mixing the state with white noise leads to a linear decrease of the results in both optimizations. For a proper amount of noise, the value of Eq. (3) will be smaller than $1 / d$ and the value of Eq. (10) strictly larger than $1 / d$. So, the state will be unfaithful, but the criterion of Observation 3(b) does not detect it. In fact, in an additional numerical analysis, we also identified one state for $d=4$ which escapes the detection as unfaithful via the criterion 3(b). This state could then be detected as unfaithful by the SDP in Eq. (10) with additional rank constraints on $\chi$ [34].

Further implications.- Among the numerous works on entanglement estimation, many approaches use the

\begin{tabular}{|c||c|c|c|c|c|}
\hline $\mathrm{d}$ & PPT & UFF [3(a)] & UFF [3(b)] & FF [3(c)] & FF [Eq. (3)] \\
\hline 3 & $0 \%$ & $25.79 \%$ & $54.68 \%$ & $45.32 \%$ & - \\
\hline 4 & $0 \%$ & $71.40 \%$ & $96.959 \%$ & $3.04 \%$ & $0.001 \%$ \\
\hline 5 & $0 \%$ & $99.33 \%$ & $99.998 \%$ & $0.002 \%$ & - \\
\hline 6 & $0 \%$ & $100 \%$ & $100 \%$ & $0 \%$ & - \\
\hline \hline
\end{tabular}

Table I. Fraction of states in the Bures metric that can be detected with the various criteria, based on a sample of $10^{6}$ states for each $d$. First, we consider the PPT states, which are always unfaithful. Then, we consider the NPT states that are unfaithful (UFF) due to Observation 3(a). Then, the NPT states that are unfaithful due to Observation 3(b). The fourth column is the fraction of states that can be detected as faithful via Observation 3(c). For $d=4$ it happened that few states were left where faithfulness could not be decided with the previous criteria. All of these states could be shown to be faithful via a direct optimization in Eq. (3) (fifth column).

comparison with the fidelity of some pure entangled states. For example, in Ref. [37] several lower bounds of entanglement measures given, which were all of the type $E(\varrho) \geq f\left[S\left(\varrho_{A B}\right)-1\right]$, where $E(\cdot)$ is some entanglement measure, $f[\cdot]$ is some function, and

$$
S\left(\varrho_{A B}\right)=\max _{|\psi\rangle}\left(\left\langle\psi\left|\varrho_{A B}\right| \psi\right\rangle / s_{1}^{2}, 1\right),
$$

which demands the maximization over all pure states. Clearly, $S>1$, if and only if $\varrho$ is faithful. From Eq. (5) it follows that for computing $S$ one needs only to optimize over maximally entangled states. Furthermore, we can conclude that $S>1$ can only happen if a state violates the CCNR criterion, and Observation 3 can also be applied to estimate $S$.

Also, note that pure states are rank-one projectors and the fidelity of a bipartite state $\varrho_{A B}$ and a pure state $|\psi\rangle$ is nothing but the expectation value $\left\langle\psi\left|\varrho_{A B}\right| \psi\right\rangle$. One can thus generalize the definition of the witness in Eq. (1) to

$$
\mathcal{W}_{V}=\varepsilon \mathbb{1}-\Pi_{V},
$$

where $\Pi_{V}$ is the projector to some subspace $V$. The minimal value of $\varepsilon$ while $\mathcal{W}$ having positive expectation value on all separable states is given by $\varepsilon_{\min }=$ $\sup _{|\psi\rangle \in V, \varrho_{\text {sep }}}\left\langle\psi\left|\varrho_{\text {sep }}\right| \psi\right\rangle$, which is the maximal value of the largest Schmidt coefficient of the pure states in $V$ [38]. With this construction, also entanglement measures can be estimated [39]. It should be noted, however, that this generalization allows the detection of some bound entangled states with positive partial transpose, e.g., if $V$ is the subspace complementary to an unextendible product basis $[12,38]$. In contrast, as we have seen, the witness in Eq. (1) can only detect entangled states with negative partial transpose. So, the witnesses in Eq. (14) are structurally quite different from fidelitybased entanglement witnesses.

Finally, one may try to extend the notion of relevant fidelity-based witnesses to the characterization of 
the Schmidt number [40, 41], this is discussed in Appendix D.

Conclusion.- The question whether the entanglement of a state can be characterized by the fidelity with some pure state is practically relevant and is closely related to the geometry of entangled states. We have provided a structural result on such faithful states for bipartite systems. For the case of two qubits, we provided a simple analytical criterion for faithfulness, and for higher dimensional systems strong necessary criteria and sufficient criteria were developed. Our results showed that fidelity-based entanglement witnesses detect a form of useful entanglement, moreover, they shed light on several other results in entanglement theory, such as the estimation of entanglement measures.

For further work, it would be very interesting to generalize the approach discussed here to multiparticle systems, where it may find applications in the certification of quantum simulation [42,43]. Also, one may consider other quantum resources (such as measurements or quantum channels) and discuss the question, which of their properties can be inferred by comparing it with a desired "pure" resource (e.g., a projective measurement or a unitary channel). This may lead to new insights into the geometry of these quantum resources.

We thank M. Navascués and M. Weilenmann for discussions. This work was supported by the Deutsche Forschungsgemeinschaft (DFG, German Research Foundation, Project No. 447948357 and No. 440958198), the Sino-German Center for Research Promotion, and the ERC (Consolidator Grant 683107/TempoQ). Y. M. acknowledges funding from a CSC-DAAD scholarship.

\section{APPENDIX A: DETAILED PROOF OF OBSERVATION 1}

In this appendix we present the detailed proof of $\mathrm{Ob}$ servation 1 for a general dimension $d$. Let us assume that the fidelity-based witness detecting $\varrho_{A B}$ is given by $\mathcal{W}=s_{1}^{2} \mathbb{1}-|\psi\rangle\langle\psi|$, with $|\psi\rangle=\sum_{i=1}^{d} s_{i}|i i\rangle$. We consider $2^{d-1}$ RFWs, coming from the maximally entangled states

$$
\left|\phi_{\vec{a}}\right\rangle=\frac{1}{\sqrt{d}}\left(|11\rangle+\sum_{j=2}^{d} a_{j}|j j\rangle\right)
$$

where $\vec{a}=\left(a_{2}, a_{3}, \ldots, a_{d}\right)$ and the coefficients $a_{j}$ can have all possible values \pm 1 . This leads to $2^{(d-1)}$ RFWs $\mathcal{W}_{\vec{a}}=\mathbb{1} / d-\left|\phi_{\vec{a}}\right\rangle\left\langle\phi_{\vec{a}}\right|$.

We aim to show that one can find probabilities $p_{\vec{a}}$ such that the operator

$$
\mathcal{Z}=\mathcal{W}-d s_{1}^{2} \sum_{\vec{a}} p_{\vec{a}} \mathcal{W}_{\vec{a}} \geq 0
$$

is positive semidefinite. This would prove already the main claim, since then $\operatorname{Tr}\left(\varrho_{A B} \mathcal{W}\right)<0$ implies that for at least one $\vec{a}$ we have $\operatorname{Tr}\left(\varrho_{A B} \mathcal{W}_{\vec{a}}\right)<0$. In order to prove Eq. (16) we aim to find $p_{\vec{a}}$ such that $\mathcal{Z}$ is diagonal. This is sufficient for positivity, as the diagonal entries of $\mathcal{Z}$ are independent of the $p_{\vec{a}}$. They are either zero or of the type $s_{1}^{2}-s_{j}^{2} \geq 0$, which ensures $\mathcal{Z} \geq 0$.

Looking at the relevant off-diagonal entries, one finds that for making $\mathcal{Z}$ diagonal, we have to find $p_{\vec{a}}$ such that

$$
\left(\begin{array}{ccccc}
\times & \alpha_{2} & \alpha_{3} & \ldots & \alpha_{d} \\
\alpha_{2} & \times & \alpha_{2} \alpha_{3} & \ldots & \alpha_{2} \alpha_{d} \\
\alpha_{3} & \alpha_{2} \alpha_{3} & \times & \ldots & \alpha_{3} \alpha_{d} \\
\vdots & \vdots & \vdots & \ddots & \vdots \\
\alpha_{d} & \alpha_{2} \alpha_{d} & \alpha_{3} \alpha_{d} & \ldots & \times
\end{array}\right)=\sum_{\vec{a}} p_{\vec{a}}\left(\begin{array}{ccccc}
\times & a_{2} & a_{3} & \ldots & a_{d} \\
a_{2} & \times & a_{2} a_{3} & \ldots & a_{2} a_{d} \\
a_{3} & a_{2} a_{3} & \times & \ldots & a_{3} a_{d} \\
\vdots & \vdots & \vdots & \ddots & \vdots \\
a_{d} & a_{2} a_{d} & a_{3} a_{d} & \ldots & \times
\end{array}\right)
$$

where we defined $\alpha_{i}=s_{i} / s_{1} \in[0,1]$ for $i=2, \ldots, d$.

With a physical argument one can see now that a decomposition as in Eq. (17) can be found: One can view the $\alpha_{i}$ as expectation values of some observables (say, $\sigma_{z}$ ) on a product state on $d-1$ qubits, i.e., a state of the form $\varrho=\varrho_{1} \otimes \varrho_{2} \otimes \cdots \otimes \varrho_{d-1}$. The terms $\alpha_{i} \alpha_{j}$ correspond then to two-body correlations $\left\langle\sigma_{z}^{(i-1)} \otimes \sigma_{z}^{(j-1)}\right\rangle$ on the same state. On the other hand, the right-hand side of Eq. (17) can be seen as a local hidden variable model, where the index $\vec{a}$ is the hidden variable occurring with probability $p_{\vec{a}}$, and the $a_{i}$ are the deterministic assign- ments for the measurement results of $\sigma_{z}$ on the different particles. For fully separable states, however, it is well known that all measurements can be explained by a local hidden variable model [22]. In fact, since one has a product state, one can directly write down the model. For that, we define

$$
p_{\vec{a}}=\prod_{j=2}^{d} q_{j}\left(a_{j}\right), \quad \text { where } \quad q_{j}( \pm 1)=\frac{1 \pm \alpha_{j}}{2} .
$$

This ensures that $q_{j}(+1)-q_{j}(-1)=\alpha_{j}$, moreover, due to the product structure, the two-body correlations are 
also reproduced.

\section{APPENDIX B: NOTES ON THE COMPLEXITY OF CHARACTERIZING FAITHFUL ENTANGLEMENT}

In this section, we will show that the problem of characterizing faithful entanglement is directly linked to the problem of so-called unitary quadratic minimization (UQM). The latter problem was recently shown to be NP-hard, as the graph 3-coloring problem can be reduced to UQM [32].

Let us start by explaining the UQM problem. We start with a set of complex $n \times n$ matrices $A_{1}, \ldots, A_{k}$ with a bounded Hilbert-Schmidt norm $\operatorname{Tr}\left(A_{j}^{\dagger} A_{j}\right) \leq 1$ for all $j=1, \ldots, k$. Then, the problem is to minimize the target function

$$
f(U)=\sum_{j=1}^{k}\left|\operatorname{Tr}\left(A_{j}^{\dagger} U\right)\right|^{2},
$$

where here and in the following $U$ always denotes an $n \times$ $n$ unitary matrix. More precisely, $\mathrm{UQM}$ is defined as a decision problem, where one is asked to decide whether either

$$
\min _{U} f(U) \leq \alpha \quad \text { or } \quad \min _{U} f(U) \geq \alpha+\frac{1}{m}
$$

where $\alpha$ is a given real number and $m$ a given positive integer. As mentioned, this problem was shown to be NP-hard [32].

In order to understand the connection to the characterization of faithful entanglement, we consider an unnormalized maximally entangled state $|\Phi\rangle=\sum_{i=1}^{d}|i i\rangle$. Then, for an arbitrary matrix $A$ we define the state

$$
\left|\Phi_{A}\right\rangle=\mathbb{1} \otimes A|\Phi\rangle .
$$

Note that any pure state can be written in this form for a suitable $A$. Furthermore, for an arbitrary unitary matrix $U$ we define

$$
\left|\Phi_{U}\right\rangle=\mathbb{1} \otimes U|\Phi\rangle
$$

The state $\left|\Phi_{U}\right\rangle$ is maximally entangled and any maximally entangled state can be written in this form. With these definitions, we have that

$$
\left\langle\Phi_{A} \mid \Phi_{U}\right\rangle=\operatorname{Tr}\left(A^{\dagger} U\right) .
$$

Applying this to Eq. (19) one finds that one can write

$$
f(U)=\left\langle\Phi_{U}|X| \Phi_{U}\right\rangle
$$

with

$$
X=\sum_{j=1}^{k}\left|\Phi_{A_{k}}\right\rangle\left\langle\Phi_{A_{k}}\right| .
$$

\begin{tabular}{|c||c|c|c|c|}
\hline $\mathrm{d}$ & PPT & UFF [3(a)] & UFF [3(b)] & FF [3(c)] \\
\hline 3 & $0.01 \%$ & $83.05 \%$ & $94.55 \%$ & $5.44 \%$ \\
\hline 4 & $0 \%$ & $99.93 \%$ & $99.999 \%$ & $0.001 \%$ \\
\hline 5 & $0 \%$ & $100 \%$ & $100 \%$ & $0 \%$ \\
\hline 6 & $0 \%$ & $100 \%$ & $100 \%$ & $0 \%$ \\
\hline \hline
\end{tabular}

Table II. Fraction of states in the Hilbert-Schmidt distribution that can be detected with the various criteria. First, we consider the PPT states, which are always unfaithful. Then, we consider the NPT states that are unfaithful (UFF) due to Observation 3(a). Then, the NPT states that are unfaithful due to Observation 3(b). The fourth column is the fraction of states that can be detected as faithful via Observation 3(c).

Now the connection to faithful entanglement becomes apparent: As we have shown in Observation 1, in order to decide faithfulness one needs to maximize the overlap with maximally entangled states. Taking $X=$ $\left(\mathbb{1}-\varrho_{A B}\right) / d$ (where the $d$ is due to the missing normalization of $\left.\left|\Phi_{U}\right\rangle\right)$ in Eqs. $(24,25)$, this leads to a minimization of $f(U)$ for a collection of $A_{j}$. Of course, one may also start from a given collection of the $A_{j}$ and then compute the corresponding $\varrho_{A B}$.

In other words, if one is able to solve the maximization problem $\max _{U}\left\langle\Phi_{U}|Y| \Phi_{U}\right\rangle$ for general hermitean operators $Y$, then one can also solve the UQM problem described above, which is known to be NP-hard. We note that the problem of deciding faithfulness is a little bit more restricted, as the operator $Y$ is normalized and one is only interested in the question whether the maximum is larger than $1 / d$ or not. Still, the argument suggests that deciding faithfulness is computationally hard.

\section{APPENDIX C: ADDITIONAL NUMERICAL RESULTS}

As mentioned in the main text, we also considered random states in the Hilbert Schmidt distribution in order to estimate which fraction is separable, entangled but unfaithful, or faithful. For two qubits we generated $10^{6}$ states and find $24.35 \%$ of all states separable via the condition of the positivity of the partial transpose (PPT) [9], and $21.14 \%$ are entangled, but unfaithful, the remaining $54.51 \%$ are faithful. As already explained in the main text, these values coincide up to statistical fluctuations with the values reported for the sufficient criterion SDP2 for unfaithfulness in Ref. [18] (or Eq. (10)). For higher dimensions, we also generated $10^{6}$ states randomly and the results are given in Table II.

\section{APPENDIX D: SCHMIDT NUMBER WITNESSES}

Here, we discuss the notion of the Schmidt number and Schmidt number witnesses. It turns out, however, 
that Observation 1 cannot so easily be generalized to this case.

\section{The concept of the Schmidt number}

The concept of entanglement and separability can be generalized to the notion of the Schmidt number. For that, one defines states to be of Schmidt number $r$, if they can be written as a convex combination of pure states with Schmidt rank $r, \varrho=\sum_{k} p_{k}\left|\phi_{k}\right\rangle\left\langle\phi_{k}\right|$, where all the states $\left|\phi_{k}\right\rangle$ have a Schmidt rank $r$. Clearly, the separable states are exactly the states with Schmidt number one.

Then, Schmidt witnesses can be defined in analogy to entanglement witnesses: A Schmidt witness for Schmidt number $\ell$ is an observable $\mathcal{S}_{\ell}$ with positive expectation value on all states with Schmidt number $\ell$. So, observing a negative expectation value proves that the state has at least Schmidt number $\ell+1$. Again, such witnesses can be based on the projector

$$
\mathcal{S}_{\ell}=\beta(\ell) \mathbb{1}-|\psi\rangle\langle\psi|,
$$

where the maximal squared overlap is now given by the sum of the $\ell$ biggest squared Schmidt coefficients,

$$
\beta(\ell)=\sum_{k=1}^{\ell} s_{k}^{2} .
$$

Again, the theory of Schmidt witnesses is well developed [38, 40,41]. The direct generalization of the RFW to the Schmidt witnesses is the set of Schmidt witnesses with $|\psi\rangle=\sum_{i=1}^{d} a_{i}|i i\rangle / \sqrt{d}$, where $a_{i}= \pm 1$. All these witnesses have $\beta(\ell)=r / d$.

\section{Fidelity-based Schmidt witnesses}

One may wonder whether a similar result as in $\mathrm{Ob}-$ servation 1 also holds for Schmidt witnesses. In the following, we show that a similar result holds for special cases, but not in general. Before going into details, we note a few general results on the order of witnesses for general convex sets.

We say a witness $\mathcal{W}$ is weaker than a set of witnesses $\left\{\mathcal{W}_{k}\right\}_{k}$, denoted as $\mathcal{W} \prec\left\{\mathcal{W}_{k}\right\}_{k}$, if for any $\varrho$ such that $\operatorname{Tr}(\mathcal{W} \varrho)<0$, there exists a $\mathcal{W}_{k}$ such that $\operatorname{Tr}\left(\mathcal{W}_{k} \varrho\right)<0$, or equivalently, if $\operatorname{Tr}\left(\mathcal{W}_{k} \varrho\right) \geq 0$ for all $k$, then $\operatorname{Tr}(\mathcal{W} \varrho) \geq 0$. This relation can be analyzed by considering the following optimization problem:

$$
\begin{aligned}
& \min : \operatorname{Tr}(\mathcal{W} \varrho) \\
& \text { subject to: } \operatorname{Tr}\left(\mathcal{W}_{k} \varrho\right) \geq 0 \text { for all } k, \\
& \varrho \geq 0
\end{aligned}
$$

More precisely, $\mathcal{W} \prec\left\{\mathcal{W}_{k}\right\}_{k}$ if and only if the solution of the optimization in Eq. (28) is zero. The dual problem of Eq. (28) reads

$$
\begin{gathered}
\max _{x_{k}}: \\
\text { subject to: } \\
\mathcal{W}-\sum_{k} x_{k} \mathcal{W}_{k} \geq 0, \\
x_{k} \geq 0 \text { for all } k .
\end{gathered}
$$

According to the general theory of convex optimization [44], weak duality gives that if there exists $x_{k} \geq 0$ such that Eq. (32) holds, then $\mathcal{W} \prec\left\{\mathcal{W}_{k}\right\}_{k}$. When the strong duality holds, e.g., in the case that $\left\{\mathcal{W}_{k}\right\}_{k}$ is a finite set, then Eq. (32) gives a necessary and sufficient condition. This can be viewed as a generalization of the result in Eq. (5).

Now, we want to investigate whether any fidelitybased Schmidt witness $\mathcal{S}_{\ell}$ defined by Eqs. (26) and (27), $\mathcal{S}_{\ell}=\beta(\ell) \mathbb{1}-|\psi\rangle\langle\psi|$ with $\beta(\ell)=\sum_{k=1}^{\ell} s_{k}^{2}$, is always weaker than the set witnesses based on maximally entangled states, i.e, whether $\mathcal{S}_{\ell} \prec\left\{\mathcal{S}_{\ell}^{\phi}\right\}$. Here, $\left\{\mathcal{S}_{\ell}^{\phi}\right\}$ denotes the set of all Schmidt number $\ell$ witnesses based on the maximally entangled state:

$$
\frac{\ell}{d} \mathbb{1}-U_{A} \otimes U_{B}\left|\phi^{+}\right\rangle\left\langle\phi^{+}\right| U_{A}^{\dagger} \otimes U_{B}^{\dagger},
$$

for all local unitary transformations $U_{A}$ and $U_{B}$. In order to see that there exists $\mathcal{S}_{\ell}$ such that $\mathcal{S}_{\ell} \nprec\left\{\mathcal{S}_{\ell}^{\phi}\right\}$, we have the following observation.

Observation 4. Let $|\psi\rangle=\sum_{i=1}^{d} s_{i}|i i\rangle$ be a pure state with Schmidt number larger than $\ell$, i.e., $s_{\ell+1}>0$. Then it can be detected by $\left\{\mathcal{S}_{\ell}^{\phi}\right\}$ if and only if $\sum_{i=1}^{d} s_{i}>\sqrt{\ell}$.

This can be seen in the following manner: When maximizing the overlap between two states over local unitaries, its best is to take them both in the same Schmidt basis [45]. So, the maximization over all maximally entangled states $|\phi\rangle$ gives

$$
\max _{|\phi\rangle}|\langle\phi \mid \psi\rangle|^{2}=\left(\sum_{i=1}^{d} \frac{s_{i}}{\sqrt{d}}\right)^{2}=\frac{1}{d}\left(\sum_{i=1}^{d} s_{i}\right)^{2} .
$$

The relation $\sum_{i=1}^{d} s_{i}>\sqrt{\ell}$ can always be satisfied when $\ell=1$. However, it can be violated when $\ell \geq 2$. On the other hand, $|\psi\rangle$ can always be detected by $\mathcal{S}_{\ell}$ defined by Eq. (26), as long as $s_{\ell+1}>0$. This implies that there exists $\mathcal{S}_{\ell}$ such that $\mathcal{S}_{\ell} \nprec\left\{\mathcal{S}_{\ell}^{\phi}\right\}$.

In general, we have the following necessary and sufficient condition for $\mathcal{S}_{\ell} \prec\left\{\mathcal{S}_{\ell}^{\phi}\right\}$ :

Observation 5. The Schmidt witness $\mathcal{S}_{\ell}$ defined in Eq. (26) satisfies that $\mathcal{S}_{\ell} \prec\left\{\mathcal{S}_{\ell}^{\phi}\right\}$ if and only if $s_{1}=s_{2}=$ $\cdots=s_{\ell}$.

The sufficiency part follows similarly to Observation 1 . To prove the necessity part, we assume that not all $s_{1}, s_{2}, \ldots, s_{\ell}$ are equal and construct a state that is 
detected by $\mathcal{S}_{\ell}$, but not by any $\mathcal{S}_{\ell}^{\phi}$. First, since not all $s_{1}, s_{2}, \ldots, s_{\ell}$ are equal, we have $\left(\sum_{i=1}^{\ell} s_{i}\right) / \sqrt{\sum_{i=1}^{\ell} s_{i}^{2}}<$ $\sqrt{\ell}$. So, there exists $0<\varepsilon \leq s_{\ell+1}$, such that

$$
\frac{1}{\sqrt{\sum_{i=1}^{\ell} s_{i}^{2}+\varepsilon^{2}}}\left(\sum_{i=1}^{\ell} s_{i}+\varepsilon\right) \leq \sqrt{\ell} .
$$

We define the state $|x\rangle=\sum_{i=1}^{\ell+1} x_{i}|i i\rangle$, with the coefficients

$$
\begin{aligned}
& x_{i}=\frac{s_{i}}{\sqrt{\sum_{i=1}^{\ell} s_{i}^{2}+\varepsilon^{2}}} \text { for } i=1,2, \ldots, \ell, \\
& x_{\ell+1}=\frac{\varepsilon}{\sqrt{\sum_{i=1}^{\ell} s_{i}^{2}+\varepsilon^{2}}},
\end{aligned}
$$

Then $|x\rangle$ cannot be detected by $\left\{\mathcal{S}_{\ell}^{\phi}\right\}$ due to Eq. (36) and Observation 4 . On the other hand, by taking advantage of the relation that $0<\varepsilon \leq s_{\ell+1}$, we have

$$
|\langle\psi \mid x\rangle|^{2}=\left(\sum_{i=1}^{\ell+1} s_{i} x_{i}\right)^{2} \geq \sum_{i=1}^{\ell} s_{i}^{2}+\varepsilon^{2}=\beta(\ell)+\varepsilon^{2} .
$$

This means that $|x\rangle$ can be detected by $\mathcal{S}_{\ell}$ but not by witnesses in the set $\left\{\mathcal{S}_{\ell}^{\phi}\right\}$.

* otfried.guehne@uni-siegen.de

$\dagger$ yuanyuan.mao@uni-siegen.de

¥ xiao-dong.yu@uni-siegen.de

[1] M. Kliesch and I. Roth, PRX Quantum 2, 010201 (2021).

[2] J. Eisert, D. Hangleiter, N. Walk, I. Roth, D. Markham, R. Parekh, U. Chabaud, and E. Kashefi, Nat. Rev. Phys. 2, 382 (2020).

[3] B. Schumacher, Phys. Rev. A 54, 2614 (1996).

[4] M. A. Nielsen, Phys. Lett. A 303, 249 (2002).

[5] O. Gühne, C.-Y. Lu, W.-B. Gao, and J.-W. Pan, Phys. Rev. A 76, 030305 (2007).

[6] S. T. Flammia and Y.-K. Liu, Phys. Rev. Lett. 106, 230501 (2011).

[7] M. Curty, M. Lewenstein, and N. Lütkenhaus, Phys. Rev. Lett. 92, 217903 (2004).

[8] L. Pezzé and A. Smerzi, Phys. Rev. Lett. 102, 100401 (2009).

[9] R. Horodecki, P. Horodecki, M. Horodecki, and K. Horodecki, Rev. Mod. Phys. 81, 865 (2009).

[10] O. Gühne and G. Tóth, Phys. Rep. 474, 1 (2009).

[11] N. Friis, G. Vitagliano, M. Malik, and M. Huber, Nat. Rev. Phys. 1, 72 (2019).

[12] B. M. Terhal, Linear Algebra Appl. 323, 61 (2000).
[13] M. Lewenstein, B. Kraus, J. I. Cirac, and P. Horodecki, Phys. Rev. A 62, 052310 (2000).

[14] G. Tóth, Phys. Rev. A 71, 010301(R) (2005).

[15] O. Gühne, M. Mechler, G. Tóth, and P. Adam, Phys. Rev. A 74, 010301 (2006).

[16] M. Piani and C. Mora, Phys. Rev. A 75, 012305 (2007).

[17] D. Chruściński and G. Sarbicki, J. Phys. A: Math. Theor. 47, 483001 (2014).

[18] M. Weilenmann, B. Dive, D. Trillo, E. A. Aguilar, and M. Navascués Phys. Rev. Lett. 124, 200502 (2020); Erratum: Phys. Rev. Lett. 125, 159903 (2020).

[19] X.-M. Hu et al., arXiv:2011.02217.

[20] G. Riccardi, D. E. Jones, X.-D. Yu, O. Gühne, and B. T. Kirby, arXiv:2102.10121.

[21] M. Bourennane, M. Eibl, C. Kurtsiefer, S. Gaertner, H.Weinfurter, O. Gühne, P. Hyllus, D. Bruß, M. Lewenstein, and A. Sanpera, Phys. Rev. Lett. 92, 087902 (2004).

[22] R. F. Werner, Phys. Rev. A 40, 4277 (1989).

[23] K. Chen and L.-A. Wu, Quantum Inf. Comput. 39, 193 (2003).

[24] O. Rudolph, Quantum Inf. Proc. 4, 219 (2005).

[25] O. Rudolph, Phys. Rev. A 67, 032312 (2003).

[26] J. de Pillis, Pacific J. Math. 23, 129 (1967).

[27] A. Jamiołkowski, Rep. Mat. Phys. 3, 275 (1972).

[28] M. Horodecki, P. Horodecki, and R. Horodecki, Phys. Rev. A 60, 1888 (1999).

[29] D. Cavalcanti, A. Acín, N. Brunner, and T. Vértesi, Phys. Rev. A 87, 042104 (2013).

[30] F. Verstraete and H. Verschelde, Phys. Rev. A 66, 022307 (2002).

[31] F. Verstraete and H. Verschelde, Phys. Rev. Lett. 90, 097901 (2003).

[32] C. D.-Y. Lee and J. Watrous, Quantum 4, 253 (2020).

[33] P. Badziag, M. Horodecki, P. Horodecki, and R. Horodecki, Phys. Rev. A 62, 012311 (2000).

[34] X.-D. Yu, T. Simnacher, H. C. Nguyen, and O. Gühne, arXiv:2012.00554.

[35] K. Życzkowski and H.-J. Sommers, J. Phys. A: Math. Gen. 34, 7111 (2001).

[36] L. J. Landau and R. F. Streater, Linear Alg. Appl. 193, 107 (1993).

[37] Y. Dai, Y. Dong, Z. Xu, W. You, C. Zhang, and O. Gühne, Phys. Rev. Applied 13, 054022 (2020).

[38] G. Sarbicki, J. Phys. A: Math. Theor. 41, 375303 (2008).

[39] K. V. Antipin, Mod. Phys. Lett. A 35, 2050254 (2020).

[40] A. Sanpera, D. Bruß, and M. Lewenstein, Phys. Rev. A 63, 050301 (2001).

[41] F. Hulpke, D. Bruß, M. Lewenstein, and A. Sanpera, Quantum Inf. Comput. 4, 207 (2004).

[42] R. D. Somma, J. Chiaverini, and D. J. Berkeland Phys. Rev. A 74, 052302 (2006).

[43] M. Gluza, M. Kliesch, J. Eisert, and L. Aolita, Phys. Rev. Lett. 120, 190501 (2018).

[44] S. Boyd and L. Vandenberghe, Convex Optimization (Cambridge University Press, 2004).

[45] O. Gühne and N. Lütkenhaus, Phys. Rev. Lett. 96, 170502 (2006). 